\title{
CASO RADIOLOGICO
}

\section{Drs. Giancarlo Schiappacasse $F^{(1)}$, Oscar Contreras $\mathbf{O}^{(2)}$, Sebastián Cabrera $V^{(3)}$.}

1. Becado de Radiología. Departamento de Radiología, Hospital Clínico Pontificia Universidad Católica de Chile.

2. Unidad Osteoarticular. Departamento de Radiología, Hospital Clínico Pontificia Universidad Católica de Chile.

3. Interno. Escuela de Medicina Pontificia Universidad Católica de Chile.

\section{Historia Clínica}

Paciente de 91 años de edad, del sexo masculino, que consulta por dolor lumbar crónico de difícil manejo médico. Tiene como antecedente de importancia una anemia crónica. Dentro de su estudio se efectuó tomografía computada de columna lumbar (Figura 1).

\section{¿Cuál es su diagnóstico?}

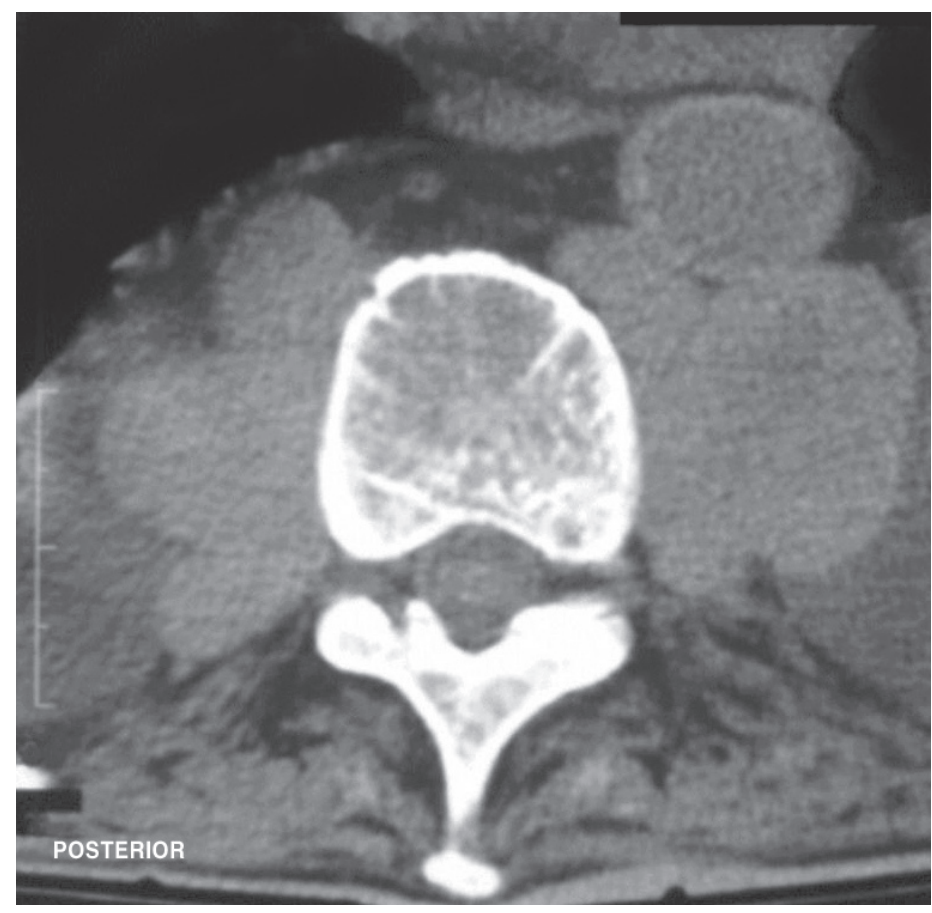

Figura 1. 


\section{RESULTADO CASO RADIOLOGICO}

\section{Drs. Giancarlo Schiappacasse $F^{(1)}$, Oscar Contreras $O^{(2)}$, Sebastián Cabrera ${ }^{(3)}$}

1. Becado de Radiología. Departamento de Radiología, Hospital Clínico Pontificia Universidad Católica de Chile.

2. Unidad Osteoarticular. Departamento de Radiología, Hospital Clínico Pontificia Universidad Católica de Chile.

3. Interno. Escuela de Medicina Pontificia Universidad Católica de Chile.

Viene de la página 5.

Diagnóstico: Hematopoyesis Extramedular

\section{Hallazgos radiológicos}

La tomografía computada (TC) de columna lumbar muestra en situación paravertebral en ambos lados (flechas) una masa polilobulada de límites bien definidos con densidad de partes blandas que no compromete los neuroforámenes y no se asocia a destrucción ósea (Figura 1).

Se efectuó punción biopsia guiada por TC de una de las masas (Figura 2), cuyo estudio histológico confirmo el diagnóstico demostrando fragmentos de tejido hematopoyético, fragmentos de tejido adiposo con extensa fibrosis colagenosa, hipocelular e infiltración linfocitaria focal (Figura 3).

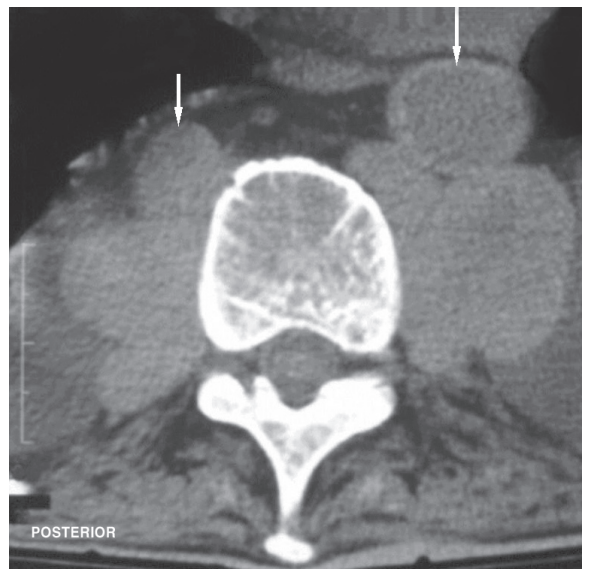

Figura 1.

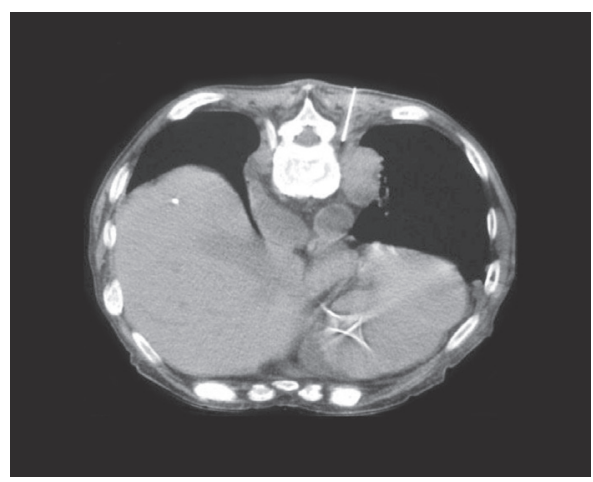

Figura 2.

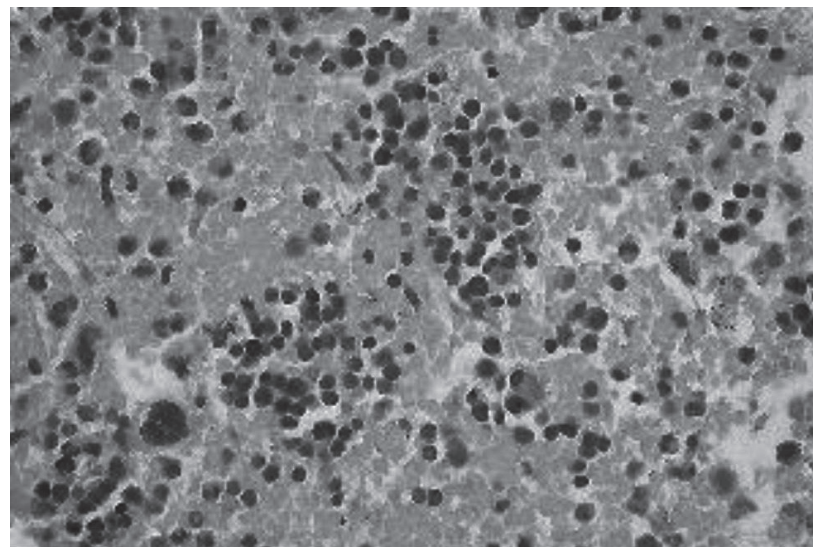

Figura 3.

\section{Discusión}

La Hematopoyesis corresponde a la formación y maduración de elementos sanguíneos, lo cual normalmente ocurre en la médula ósea de huesos largos, costillas y en las vértebras de los adultos. En la vida fetal los principales sitios de hematopoyesis se encuentran en el saco vitelino, bazo e hígado.

Cuando los sitios primarios de hematopoyesis fallan en la vida adulta, como ocurre en algunas patologías como la mielofibrosis y algunas hemoglobinopatías, especialmente B-talasemias y enfermedad de células falciformes, diversos sitios extramedulares asumen la formación de los elementos sanguíneos.

Esta última se observa principalmente en sitios como el hígado, bazo y regiones paraespinales del tórax y abdomen, siendo más frecuente en la región torácica inferior. Sin embargo, este proceso puede comprometer virtualmente cualquier órgano o tejido constituyendo focos de hematopoyesis extramedular (HE).

La HE en general es asintomática y sólo ocasionalmente pueden existir molestias dadas por efecto de masa.

Los hallazgos radiológicos más comunes lo constituyen masas paraespinales y expansión de las costillas. Las masas son bien delimitadas y en TC con contraste presentan una leve captación 
homogénea. Se ubican con frecuencia rodeando las cadenas simpáticas paravertebrales, localización en que es frecuente observar tumores neurogénicos. Con menor frecuencia los elementos hematopoyéticos se ubican en el espacio precardíaco, pleural y también en el intersticio pulmonar simulando un proceso intersticial neoplásico o inflamatorio.

El tejido paraespinal hematopoyético puede extenderse al canal espinal causando síntomas de tipo neurológicos secundarios a compresión.

Dentro de los diagnósticos diferenciales más relevantes, en presencia de estas masas paravertebrales, se deben considerar los tumores neurogénicos tales como: ganglioneuroma, neurinoma, neurofibroma, abscesos paraespinales (TBC), meningoceles laterales y linfoma.

La HE puede ser tratada con transfusiones frecuentes para disminuir el estimulo hematopoyético; en forma asociada se ha visto que la terapia con hidroxiurea reduce el tamaño de las masas con lo que alivia los síntomas compresivos.
Este caso constituye una causa poco frecuente de masa paraespinal cuyo diagnóstico requiere de sospecha clínica y un reconocimiento del aspecto imagenológico. Sin embargo, el diagnóstico final debe de ser confirmado mediante biopsia y estudio histológico de la lesión.

\section{Bibliografía}

1. Christos S. Geogiades, Edward G.Neyman, et al. Typical and atypical presentations of extramedullary hemopoiesis. AJR 2002; 179: 1239-1243.

2. Krouwels F.H, Bresser P, von de Borne AE. Extramedullary Hematopoiesis: Breathtaking and HairRaising. N England J Med 1999; 341: 1702-1704.

3. Tsitouridis J, Stamos S, Hassapopoulou E et al. Extramedullary paraspinal hematopoiesis in thalassemia: CT and MRI evaluation. Eur $\mathrm{J}$ Radiol 1999; 30: 33-38.

Correspondencia: Dr. Giancarlo Schiappacasse F. E-mail:gschf@vtr.cl 